\title{
Nonreciprocal transmission of multi-band optical signals in thermal atomic systems
}

\author{
Shengfa Fan (范圣法 $)^{1,2}$, Yihong Qi (祁义红) $)^{1 *}$, Yueping Niu (钮月萍) ${ }^{1}$, and Shangqing Gong (龚尚庆) ${ }^{1}$ \\ ${ }^{1}$ School of Physics, East China University of Science and Technology, Shanghai 200237, China \\ ${ }^{2}$ School of Materials Science and Engineering, East China University of Science and Technology, Shanghai 200237, China
}

*Corresponding author: qiyihong@ecust.edu.cn

Received June 14, 2021 | Accepted September 6, 2021 | Posted Online November 8, 2021

\begin{abstract}
Multi-band signal propagation and processing play an important role in quantum communications and quantum computing. In recent years, optical nonreciprocal devices such as an optical isolator and circulator are proposed via various configurations of atoms, metamaterials, nonlinear waveguides, etc. In this work, we investigate all-optical controlled nonreciprocity of multi-band optical signals in thermal atomic systems. Via introducing multiple strong coupling fields, nonreciprocal propagation of the probe field can happen at some separated frequency bands, which results from combination of the electromagnetically induced transparency (EIT) effect and atomic thermal motion. In the proposed configuration, the frequency shift resulting from atomic thermal motion takes converse effect on the probe field in the two opposite directions. In this way, the probe field can propagate almost transparently within some frequency bands of ElT windows in the opposite direction of the coupling fields. However, it is well blocked within the considered frequency region in the same direction of the coupling fields because of destruction of the EIT. Such selectable optical nonreciprocity and isolation for discrete signals may be greatly useful in controlling signal transmission and realizing selective optical isolation functions.
\end{abstract}

Keywords: optical nonreciprocity; atomic thermal motion; electromagnetically induced transparency; multi-band. DOI: 10.3788/COL202220.012701

\section{Introduction}

Similar to their electronic counterpart, optical nonreciprocal devices play an important role and possess the fundamental function in photonic devices and quantum circuits, urging great research interest in an optical isolator ${ }^{[1-3]}$, circulator $^{[4-6]}$, and router ${ }^{[7,8]}$. These optical nonreciprocal devices allow photon transportation in one direction, while blocking it in the reverse direction. In laser systems, the optical isolator, which blocks the back transmission of light, provides effective protection for the lasers. Optical nonreciprocal devices also have promising applications in all-optical quantum networking and computing. Transmission and processing of multi-wavelength/band optical signals is an important issue in optical communications and processing. Optical devices for multi-wavelength applications have attracted intensive attention in recent years, such as multi-wavelength lasers ${ }^{[9-13]}$, multiplexing and communications $s^{[4,15]}$, imaging and sensors ${ }^{[16-19]}$, and photovoltaic devices $^{[20]}$. It is also a very important subject to develop and design optical nonreciprocal devices such as optical isolators suitable for multi-wavelength applications ${ }^{[21,22]}$. In this work, we are committed to the study of controllable multi-band optical nonreciprocal transmission by using atomic systems.
The key to realize optical nonreciprocal devices is the nonreciprocal or asymmetric transmission of light. Magneto-optical materials were used to produce optical nonreciprocal propagation via the Faraday rotation effect ${ }^{[23-27]}$. However, responses of the magnetic materials are often very weak, implying requirements of the large size bulk magnetic media or the strong magnetic fields, which may bring about some unfavorable impacts ${ }^{[1]}$. Then, for avoiding the use of magnetic materials, schemes based on optical nonlinearity ${ }^{[28-31]}$ or photonic crystal heterojunctions ${ }^{[32]}$ were proposed. Recently, a number of works dedicated to realizing nonmagnetic optical nonreciprocal transmission via different schemes, such as frequency conversion ${ }^{[33,34]}$, angular momentum biasing ${ }^{[35-37]}$, optoacoustic effects ${ }^{[34,38]}$, artificial gauge field ${ }^{[39-41]}$, parity-time-symmetry breaking ${ }^{[22-45]}$, and moving medium ${ }^{[46,47]}$.

Generally, random thermal motion of atoms has a negative impact on coherence of the quantum system, resulting in the decoherence effect or thermal noise ${ }^{[48-50]}$. It is fortunate that via a smart design people can also actively utilize the atomic thermal motion in some particular fields. Based on the electromagnetically induced transparency (EIT) effect and the thermal motion of atoms, Zhang et al. proposed a novel mechanism to 
achieve optical propagation of the probe field in a three-level $\Lambda$ type atom-cavity coupling system ${ }^{[51]}$. Then, Xia et al. investigated the direction-dependent cross phase modulation (XPM) in an N-type thermal atomic system and utilized the XPM to achieve an optical isolator and circulator ${ }^{[52]}$. Later, Gong et al. also investigated directional optical amplification ${ }^{[53]}$, optical isolation by the pumping effect ${ }^{[54]}$, and broadband optical nonreciprocity $^{[55]}$ in multi-level atomic systems. Manipulation of multiple optical signals may have potential applications in optical communications and quantum information processing. Utilizing dynamically induced photonic band gaps, Yang et al. have proposed a scheme to generate two-color optical nonreciprocity in a cold tripod-type atomic system ${ }^{[56]}$. In this work, stimulated by these works, we investigated all-optical controlled nonreciprocal propagation of multi-band optical signals in a Ytype-like multi-level hot atomic system. By introducing multiple strong coupling fields, some nonreciprocal bands with separated frequencies can be generated for the weak probe field. Moreover, these separated nonreciprocal frequency bands can be flexibly controlled by the coupling fields. This work may have potential applications in multi-band signal detection, discrimination, and processing.

\section{Model and Equations}

We consider interaction of laser fields and the atomic system of $N+2$ levels, as shown in Fig. 1, in which the weak probe field and $N$ strong coupling fields couple corresponding energy levels in Fig. 1(a) and propagate along different directions, respectively, as shown in Figs. 1(b) and 1(c). The weak probe field $\Omega_{p}$ of frequency $\omega_{p}$ couples states $|\mathrm{g}\rangle$ and $|\mathrm{m}\rangle$, and the transitions $|\mathrm{m}\rangle \leftrightarrow|n\rangle(n=1, \ldots, N)$ are driven by the strong coupling fields $\Omega_{n}$ of frequency $\omega_{n}$, where $\Omega_{p}=\left|\mu_{\mathrm{gm}}\right| E_{p} / 2 \hbar$ and $\Omega_{n}=$ $\left|\mu_{\mathrm{m} n}\right| E_{n} / 2 \hbar$ are corresponding half-Rabi frequencies of the fields with the electric dipole momentum $\mu_{i j}(i, j=\mathrm{g}, \mathrm{m}, 1, \ldots, N)$ and the electric field amplitudes $E_{p}$ and $E_{n}$. The atomic gas is loaded in a cell, and its temperature is controlled by a temperature control system. In general, the atoms are in constant thermal motion following the Maxwell velocity distribution. Under

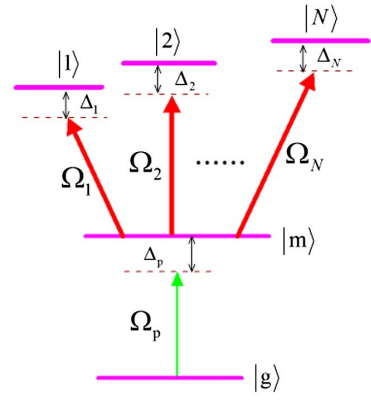

(a)

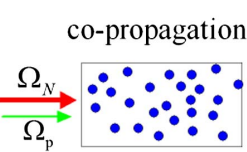

(b)

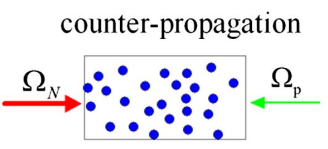

(c)
Fig. 1. Interaction of the laser fields and the multi-level atomic systems. (a) Laser coupling scheme, (b) co-propagation, and (c) counter-propagation setups of the probe field and the strong coupling fields. the electric dipole and rotating-wave approximations, the Hamiltonian of the system can be written in the interaction picture as

$$
\begin{aligned}
H_{\text {int }}= & -\hbar\left[\Delta_{p}|\mathrm{~m}\rangle\left\langle\mathrm{m}\left|+\sum_{n=1}^{N}\left(\Delta_{p}+\Delta_{n}\right)\right| n\right\rangle\langle n|\right. \\
& \left.+\left(\Omega_{p}|\mathrm{~m}\rangle\left\langle\mathrm{g}\left|+\sum_{n=1}^{N} \Omega_{n}\right| n\right\rangle\langle\mathrm{m}|+\text { H.C. }\right)\right]
\end{aligned}
$$

where $\Delta_{p}=\bar{\omega}_{\mathrm{mg}}-\omega_{p}\left(\Delta_{n}=\bar{\omega}_{n \mathrm{~m}}-\omega_{n}\right)$ denotes the detuning of the probe field (coupling fields) for the corresponding transition $|\mathrm{g}\rangle \leftrightarrow|\mathrm{m}\rangle(|\mathrm{m}\rangle \leftrightarrow|n\rangle)$ with transition frequency $\bar{\omega}_{\mathrm{mg}}\left(\bar{\omega}_{n \mathrm{~m}}\right)$. From the Liouville equation, we can obtain the following motion equations for the density-matrix elements:

$$
\dot{\rho}_{\mathrm{gg}}(t)=i \Omega_{p} \rho_{\mathrm{mg}}(t)-i \Omega_{p}^{*} \rho_{\mathrm{gm}}(t)+\sum_{n=1}^{N} \Gamma_{n g} \rho_{n n}(t)+\Gamma_{\mathrm{mg}} \rho_{\mathrm{mm}}(t),
$$

$$
\begin{aligned}
\dot{\rho}_{\mathrm{mm}}(t)= & i \Omega_{p} \rho_{\mathrm{gm}}(t)+i \sum_{n=1}^{N}\left[\Omega_{n} \rho_{n \mathrm{~m}}(t)-\Omega_{n}^{*} \rho_{n \mathrm{~m}}(t)\right] \\
& -i \Omega_{p}^{*} \rho_{\mathrm{mg}}(t)-\Gamma_{\mathrm{mg}} \rho_{\mathrm{mm}}(t)+\sum_{n=1}^{N} \Gamma_{n \mathrm{~m}} \rho_{n n}(t),
\end{aligned}
$$

$$
\begin{gathered}
\dot{\rho}_{n n}(t)=i \Omega_{n}^{*} \rho_{\mathrm{m} n}(t)-i \Omega_{n} \rho_{n \mathrm{~m}}(t)-\Gamma_{n \mathrm{~m}} \rho_{n n}(t)-\Gamma_{n g} \rho_{n n}(t), \\
\dot{\rho}_{\mathrm{mg}}(t)=\left(i \Delta_{p}-\gamma_{\mathrm{mg}}\right) \rho_{\mathrm{mg}}(t)+i \sum_{n=1}^{N} \Omega_{n} \rho_{n \mathrm{~g}}(t) \\
+i \Omega_{p}^{*} \rho_{\mathrm{gg}}(t)-i \Omega_{p}^{*} \rho_{\mathrm{mm}}(t),
\end{gathered}
$$

$\dot{\rho}_{n \mathrm{~g}}(t)=\left(i \Delta_{p}+i \Delta_{n}-\gamma_{n \mathrm{~g}}\right) \rho_{n \mathrm{~g}}(t)+i \Omega_{n}^{*} \rho_{\mathrm{mg}}(t)-i \Omega_{p}^{*} \rho_{n \mathrm{~m}}(t)$,

$$
\begin{aligned}
\dot{\rho}_{n \mathrm{~m}}(t)= & i\left(\Delta_{n}+i \gamma_{n \mathrm{~m}}\right) \rho_{n \mathrm{~m}}(t)+i \Omega_{n}^{*} \rho_{\mathrm{mm}}(t) \\
& -i \Omega_{p} \rho_{n \mathrm{~g}}(t)-i \sum_{l=1}^{N} \Omega_{l}^{*} \rho_{n l}(t), \\
\dot{\rho}_{n l}(t)= & i\left(\Delta_{n}-\Delta_{l}+i \gamma_{n l}\right) \rho_{n l}(t)+i \Omega_{n} \rho_{\mathrm{m} l}(t) \\
& -i \Omega_{l}^{*} \rho_{n \mathrm{~m}}(t), \quad n \neq l,
\end{aligned}
$$

with $\sum_{n=1}^{N} \rho_{n n}(t)+\rho_{\mathrm{gg}}(t)+\rho_{\mathrm{mm}}(t)=1, \quad \rho_{i j}(t)=\rho_{j i}^{*}(t)(i, j=$ $\mathrm{g}, \mathrm{m}, n ; i \neq j$ ), and $n, l=1-N$. We denote the radiative decay rate of the populations from level $|n\rangle$ to $|\mathrm{m}\rangle(|\mathrm{g}\rangle)$ by $\Gamma_{n \mathrm{~m}}\left(\Gamma_{n \mathrm{~g}}\right)$ and the decoherence rate by $\gamma_{n l}$, respectively. Assuming $\Omega_{p}<\Omega_{n}, \Gamma_{i j}$, the atoms are mainly populated on state $|\mathrm{g}\rangle$. Then, by solving the density matrix of Eqs. (2)-(8) in steady state, we can obtain the linear susceptibility for the weak probe field as 


$$
\chi \approx \frac{-\mathcal{N}_{D}\left|\mu_{\mathrm{gm}}\right|^{2}}{\Delta_{p}+i \gamma_{\mathrm{mg}}-\sum_{n=1}^{N} \Omega_{n}^{2} /\left(\Delta_{p}+\Delta_{n}+i \gamma_{n \mathrm{~g}}\right)},
$$

with the atom density $\mathcal{N}_{D}$.

Due to the irregular thermal motion, atoms in the hot atomic system move with various velocities in different directions. Under this condition, both frequencies of the lasers and frequency shift arising from the Doppler effect take effect on the interaction between lasers and atoms. Then, the detuning $\Delta_{i}(i=p, n)$ in Eq. (9) should be rewritten as $\Delta_{i} \pm k_{i} v_{j}$ with the atom of velocity $v_{j}$ and the wavevector $k_{i}$ of the laser $\Omega_{i}$. Assuming all the coupling lasers $\Omega_{n}$ propagate along the same direction, the effective macro susceptibility for the probe field $\Omega_{p}$ of co-/counter-propagation with the coupling lasers should be integrated on all the atoms of different velocities by

$$
\chi_{\mathrm{co}(\mathrm{cou})}=\int_{-\infty}^{+\infty} \chi D(v) \mathrm{d} v,
$$

in which $D(v)=e^{-\frac{v^{2}}{v_{p}^{2}}} /\left(\sqrt{\pi} v_{p}\right)$ indicates the Maxwell-Boltzmann distribution of the atoms, and $v_{p}=\sqrt{2 k_{\mathrm{B}} T / M}$ is the most probable velocity with the Boltzmann constant $k_{\mathrm{B}}$, the absolute temperature $T$, and the atom mass $M$. Then, susceptibilities for the co-/counter-propagating probe field can be obtained via the following integrations:

$$
\begin{aligned}
\chi_{\mathrm{co}} & =\int_{-\infty}^{+\infty} \frac{-\mathcal{N}_{D}\left|\mu_{\mathrm{gm}}\right|^{2} D(v)}{\Delta_{p}-k v+i \gamma_{\mathrm{mg}}-S_{1}} \mathrm{~d} v, \\
\chi_{\mathrm{cou}} & =\int_{-\infty}^{+\infty} \frac{-\mathcal{N}_{D}\left|\mu_{\mathrm{gm}}\right|^{2} D(v)}{\Delta_{p}+k v+i \gamma_{\mathrm{mg}}-S_{2}} \mathrm{~d} v,
\end{aligned}
$$

with

$$
\begin{aligned}
& S_{1}=\sum_{n=1}^{N} \Omega_{n}^{2} /\left(\Delta_{p}+\Delta_{n}-2 k v+i \gamma_{n \mathrm{~g}}\right), \\
& S_{2}=\sum_{n=1}^{N} \Omega_{n}^{2} /\left(\Delta_{p}+\Delta_{n}+i \gamma_{n \mathrm{~g}}\right),
\end{aligned}
$$

where we have assumed $k_{p} \approx k_{i}=k(i=1, \ldots, N)$ for simplicity. Transmission of the probe field in the medium can be obtained from the Maxwell equations as follows:

$$
t_{\mathrm{co}(\mathrm{cou})}=e^{i \alpha \chi_{\mathrm{co}(\mathrm{cou})} L},
$$

with the transmission coefficient $\alpha=\frac{\omega_{p}}{2 \varepsilon_{0} \hbar c}$ and medium length $L$. Then, the normalized transmissivity for the probe field is

$$
T_{\text {co(cou) }}=\left|t_{\text {co(cou) }}\right|^{2} .
$$

So, transmission of the probe field in the co-/counterpropagation direction can be regulated to pursue high asymmetric transmission in the two opposite directions by controlling the coupling fields.

\section{Results and Discussion}

Figure 2 shows transmissions of the co-propagating (red dashdotted line) and the counter-propagating (blue solid line) probe fields in the three, four, five, and six-level atomic systems. In the calculation, we consider the atomic medium length $L=5.0 \mathrm{~cm}$, the atomic density $N_{D}=5.0 \times 10^{16} \mathrm{~m}^{-3}$, the temperature $T=70.0^{\circ} \mathrm{C}$, and the other parameters are normalized by $\gamma=\gamma_{\mathrm{mg}}$. It is clear that multiple nonreciprocal windows with separated frequency bands are generated in the multi-level atomic systems. When the probe field propagates along the opposite direction of the coupling fields, effects of the atom motion on the probe field and the coupling fields can be offset in the proposed configuration, which leads to the construction of the EIT under two-photon resonance $\left(\Delta_{p}+\Delta_{n}=0\right)$ and thus high transmissivity of the probe field in the EIT windows. Then, it can be seen that one or several separated high transmission bands (blue solid lines) are created in the transmission spectrum for the probe field, depending on the number and detuning of control fields. However, for the co-propagating probe field, frequency shifts induced by the atom motion produce remarkable two-photon detunings for the probe field and the coupling fields, which destruct the EIT effect and make large absorption of the probe field. Under this condition, the weak probe field interacts with an effective two-level atomic system, and probe photons are greatly absorbed by a large number of atoms. The following spontaneous emission can never generate a field along the incident direction of the probe field. Transmission of the co-propagating probe field is almost vanishing
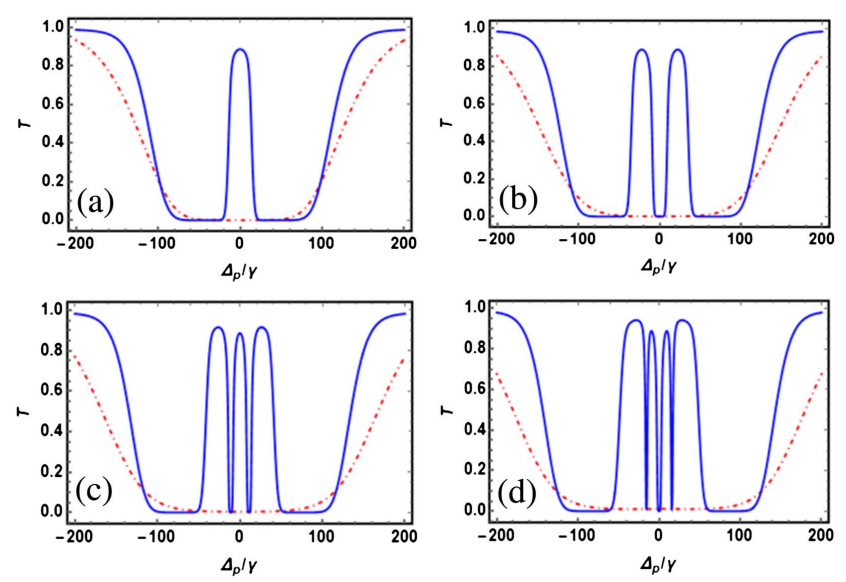

Fig. 2. Transmission of the probe field in multi-level atomic systems as a function of the probe detuning $\Delta_{p}$, where the blue solid line stands for the counter-propagation and the red dash-dotted line for co-propagation. (a) Three-level system with $\Delta_{1}=0, \Omega_{1}=40 \gamma_{\text {i }}$ (b) four-level system with $\Delta_{1}=-20 \gamma_{1} \Delta_{2}=20 \gamma_{1} \Omega_{1}=\Omega_{2}=40 \gamma_{i}$ (c) five-level system with $\Delta_{1}=-20 \gamma_{1}$ $\Delta_{2}=0, \Delta_{3}=20 \gamma_{1} \Omega_{1}=\Omega_{2}=\Omega_{3}=40 \gamma_{1}$ and (d) six-level system with $\Delta_{1}=-20 \gamma_{1} \Delta_{2}=-10 \gamma_{1} \Delta_{3}=10 \gamma_{1} \Delta_{4}=20 \gamma_{1} \Omega_{1}=\Omega_{2}=\Omega_{3}=\Omega_{4}=40 \gamma$. 
(red dashed-dotted lines) in a wide spectrum range. Therefore, multi-band nonreciprocal propagation of the probe field can be achieved in this atomic system by introducing multiple coupling lasers driving corresponding transitions.

In this scheme, each band for nonreciprocal propagation of light can be well controlled and shifted individually by changing the detuning of the corresponding coupling field, which brings us great convenience for optical signal or information processing. Figure 3 shows the transmissions of the co-propagating and counter-propagating probe fields with different detunings of the coupling fields in the five-level atomic system. It can be seen in Figs. 3(a)-3(c), with the fixed detunings $\Delta_{1}$ and $\Delta_{3}$, the central frequency of the middle nonreciprocal band is shifted independently by tuning $\Delta_{2}$. Clearly, the left and right nonreciprocal bands can also be controlled by changing $\Delta_{1}$ and $\Delta_{3}$, respectively. Such frequency-tunable multi-band nonreciprocity may be very helpful in the processing of optical multi-band signals.

Bandwidth of optical nonreciprocal devices plays an important role in applications ${ }^{[57,58]}$. Broad and tunable widths of nonreciprocal windows are often desirable for nonreciprocal devices such as an optical isolator and circulator. In this scheme, these nonreciprocal windows can be controlled individually or simultaneously by adjusting intensities of the coupling fields. To further examine the dependence of transmissivity and nonreciprocal bandwidth of the probe field on Rabi frequencies of the coupling fields, we calculated transmissions of co-propagating and counter-propagating probe fields in the five-level atomic system by changing the Rabi frequencies of the coupling fields. In Figs. 4(a) and 4(b), for simplicity, we adjust simultaneously the Rabi frequencies of the coupling fields by $\Omega_{1}=$ $\Omega_{2}=\Omega_{3}=\Omega_{0}$. It is shown that, for the counter-propagating probe field, bandwidths of the central three separated high transmission bands increase simultaneously with the Rabi frequencies of the coupling fields, while transmission of the copropagating probe field is well suppressed in corresponding frequency windows, and the total width of the absorption

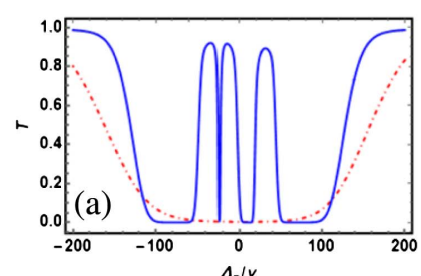

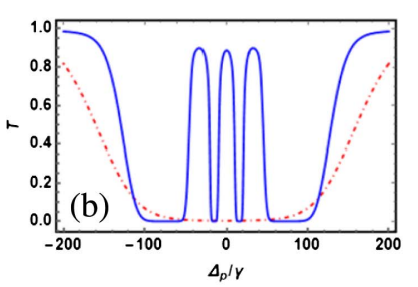

$\Delta_{p} / \gamma$

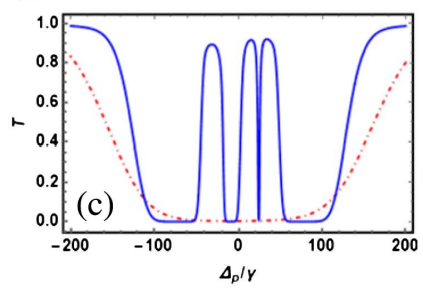

Fig. 3. Tunable nonreciprocal frequency bands in the five-level atomic system with $\Delta_{2}=0, \Omega_{1}=\Omega_{2}=\Omega_{3}=40 \gamma_{1} \Delta_{1}=-30 \gamma_{1} \Delta_{3}=30 \gamma$ and (a) $\Delta_{2}=20 \gamma_{1}$ (b) $\Delta_{2}=$ $0 \gamma_{1}$ (c) $\Delta_{2}=-20 \gamma$. The other parameters are the same as in Fig. 2 .
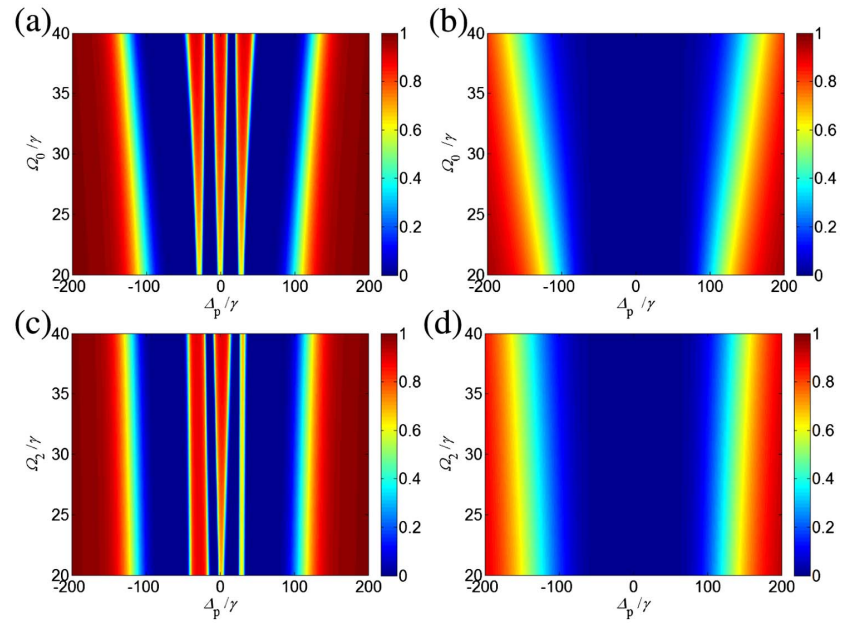

Fig. 4. Variation of transmission of probe fields with (a), (b) $\Delta_{p}$ and $\Omega_{0}$ or (c), (d) $\Omega_{2}$, where (a), (c) are the results for the counter-propagating probe field and (b), (d) for the co-propagating probe field. In the calculation, $\Delta_{1}=-30 \gamma_{\text {, }}$ $\Delta_{2}=0, \Delta_{3}=30 \gamma_{1}$ and the other parameters are the same as in Fig. 2 .

window is also broadened with the Rabi frequency of the coupling fields. In Figs. 4(c) and 4(d), we fix $\Omega_{1}=40 \gamma$ and $\Omega_{3}=20 \gamma$ and only change $\Omega_{2}$ to control transmission of the probe field. In this case, only the bandwidth of the central nonreciprocal window is enlarged with the increase of $\Omega_{2}$, but the left and right ones are almost unchanged.

To further examine transmissivity and contrast $\eta=\left|\frac{T_{\mathrm{cou}}-T_{\mathrm{co}}}{T_{\mathrm{cou}}+T_{\mathrm{co}}}\right|$ for the case of Figs. 3(c) and 3(d), we calculate and plot the transmissivity and corresponding transmission contrast at the central frequencies of the three nonreciprocal bands in Fig. 5. As shown in Fig. 5(a), transmission of the probe field at $\Delta_{p}=0$ is enhanced obviously with the increase of $\Omega_{2}$, while at the other two nonreciprocal bands the probe fields have little change. This provides us with a way to flexibly control transmission of signals in need in the nonreciprocal windows. It is anticipated that high transmissions of the probe fields at different nonreciprocal bands can be achieved via increasing the corresponding intensities of the coupling fields. Figure 5(b) shows high transmission contrasts at the center of the three nonreciprocal bands, implying excellent isolation performance of them. (a)

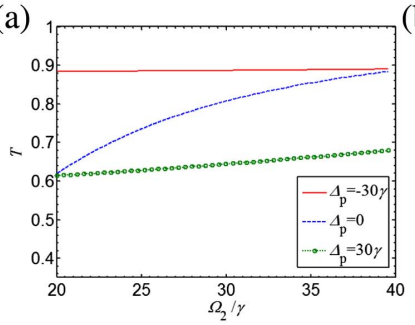

b)

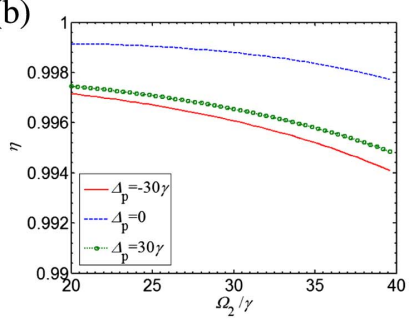

Fig. 5. Variations of (a) transmissivity $T$ of the counter-propagating probe field and (b) corresponding transmission contrast $\eta$ with Rabi frequency of the coupling field $\Omega_{2}$ at the center frequencies of the three nonreciprocal bands, corresponding to the cases of Figs. 4(c) and 4(d). 


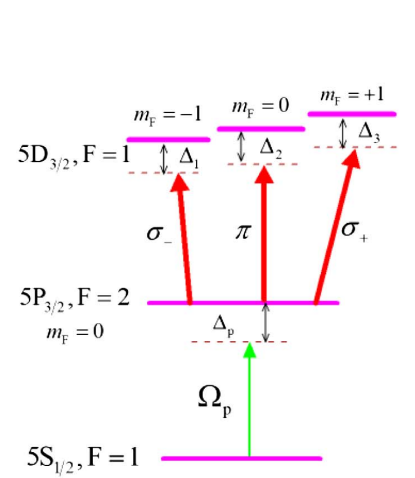

(a)

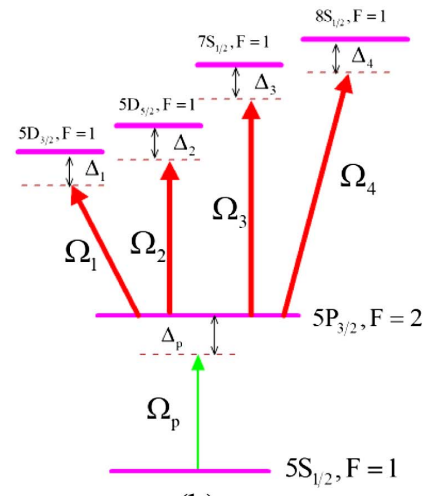

(b)
Fig. 6. Possible atomic systems and laser coupling schemes in experiments, where probe and control fields are with (a) adjacent frequencies by using Zeeman splitting levels and (b) different frequencies.

It is necessary to have further discussion on the experimental feasibility and possible atomic systems. In the calculation, we have assumed $k_{p} \approx k_{i}$ for simplicity, where the effect for the Doppler shift can be well canceled for the probe field in the counter-propagating directions. Generally speaking, it is not easy to find proper atomic transitions. However, Zeeman splitting levels in an alkali metal atomic system such as rubidium and cesium can provide a feasible way for realizing this model. For example, we can choose the transition $5 \mathrm{~S}_{1 / 2}, F=1 \rightarrow$ $5 \mathrm{P}_{3 / 2}, F=2(384.23034 \mathrm{THz})$ as the probe field coupled levels and make the control fields with different polarizations couple the transitions $5 \mathrm{P}_{3 / 2}, F=2\left(m_{F}=0\right) \rightarrow 5 \mathrm{D}_{3 / 2}, F=1\left(m_{F}= \pm 1,0\right)$ (386.25231 THz) in Rb-87 atoms [as shown in Fig. 6(a)]. Then, in similar configurations, it can be greatly guaranteed that $k_{p} \approx k_{i}$. Even for $k_{p} \neq k_{i}$, the absorption can still be largely reduced in the counter-propagating direction, and thus nonreciprocity forms due to the partially eliminated Doppler effect and Doppler broadened linewidth of thermal atoms. For example, we can choose the atomic system and the laser coupling scheme, as shown in Fig. 6(b), where the probe laser couples the transition $5 \mathrm{~S}_{1 / 2}, F=1 \rightarrow 5 \mathrm{P}_{3 / 2}, F=2$, and the control

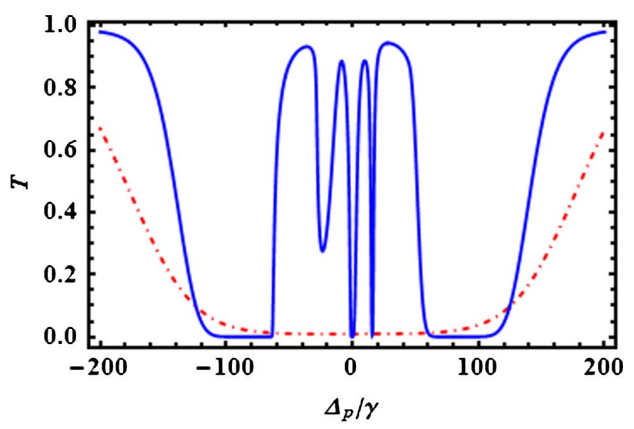

Fig. 7. Transmission of the probe field in counter-propagating (blue solid line) and co-propagating (red dashed line) directions by using the scheme in Fig. 6(b), where $\Delta_{1}=-40 \gamma_{1} \Delta_{2}=-10 \gamma_{1} \Delta_{3}=10 \gamma_{1} \Delta_{4}=40 \gamma$, and other parameters are the same as in Fig. 2(d). laser couples the transitions $5 \mathrm{P}_{3 / 2}, F=2 \rightarrow 5 \mathrm{D}_{3 / 2}, F=1$ $\left(5 \mathrm{D}_{5 / 2}, F=1 ; 7 \mathrm{~S}_{1 / 2}, F=1 ; 8 \mathrm{~S}_{1 / 2}, F=1\right)$. In this case, $\omega_{p}=2 \pi \times$ $384.23034 \mathrm{THz}$ and $\omega_{1,2,3,4}=2 \pi \times(386.25231,386.3411$, $404.5667,486.58499) \mathrm{THz}$ are used for calculation. It can be found that, as long as $k_{p}$ and $k_{i}$ are not too different, the property of nonreciprocity can be well kept. The only difference is that the transmission of the probe field in the counter-propagating direction may be suppressed slightly, or part of the multi-band signals cannot be well separated (as shown in Fig. 7). Therefore, multiband nonreciprocity can also be achieved by using similar multilevel transitions in alkali-metal atoms, such as rubidium and cesium. For example, transitions of $\left(5 \mathrm{~S}_{1 / 2}\right) \leftrightarrow\left(5 \mathrm{P}_{1 / 2}, 5 \mathrm{P}_{3 / 2}\right) \leftrightarrow$ $\left(6 \mathrm{~S}_{1 / 2}, 7 \mathrm{~S}_{1 / 2}, 8 \mathrm{~S}_{1 / 2}, \ldots, 5 \mathrm{D}_{3 / 2}, 6 \mathrm{D}_{3 / 2}, 7 \mathrm{D}_{3 / 2}, 8 \mathrm{D}_{3 / 2} \ldots\right)$ in rubidium provide the possibility of cascade-like transitions. In addition, small tilt angles between the probe and coupling fields may also be arranged for matching the condition of $k_{p} \approx k_{i}$ in experiment.

\section{Conclusions}

In conclusion, based on the EIT effect, we have investigated controllable multi-band nonreciprocal propagation of optical signals in the thermal multi-level cascade atomic systems. By use of multiple strong coupling fields, the weak probe field can propagate with several separated high transmission bands in the opposite direction of the coupling fields due to the EIT effect, while the co-propagating probe field can be well absorbed in the same frequency domain. This provides the possibility of generating and flexibly controlling multi-band nonreciprocal propagation of optical signals. Moreover, separation, bandwidth, and center frequencies of these nonreciprocal transmission bands can be well adjusted and controlled by changing the Rabi frequencies and detunings of the coupling lasers. Simultaneously, high transmission contrast can be maintained in these nonreciprocal bands, guaranteeing excellent optical isolation performance. This work may provide references for related optical isolation devices such as an optical diode and circulator. Other probable functions of the separated nonreciprocal bands may be extracting and discriminating optical signals, which may find application in optical information processing and optical networking.

\section{Acknowledgement}

This work was supported by the National Natural Science Foundation of China (Nos. 11874146, 11974109, and 12034007).

\section{References}

1. K. Xia, G. Lu, G. Lin, Y. Cheng, Y. Niu, S. Gong, and J. Twamley, "Reversible nonmagnetic single-photon isolation using unbalanced quantum coupling," Phys. Rev. A 90, 043802 (2014). 
2. F. Ruesink, M.-A. Miri, A. Alù, and E. Verhagen, "Nonreciprocity and magnetic-free isolation based on optomechanical interactions," Nat. Commun. 7, 13662 (2016)

3. F. Ruesink, J. P. Mathew, M.-A. Miri, A. Alù, and E. Verhagen, "Optical circulation in a multimode optomechanical resonator," Nat. Commun. 9, 1798 (2018).

4. M. Scheucher, A. Hilico, E. Will, J. Volz, and A. Rauschenbeutel, "Quantum optical circulator controlled by a single chirally coupled atom," Science 354, 1577 (2016).

5. X. W. Xu and Y. Li, "Optical nonreciprocity and optomechanical circulator in three-mode optomechanical systems," Phys. Rev. A 91, 053854 (2015).

6. K. Fang, J. Luo, A. Metelmann, M. H. Matheny, F. Marquardt, A. A. Clerk, and O. Painter, "Generalized nonreciprocity in an optomechanical circuit via synthetic magnetism and reservoir engineering," Nat. Phys. 13, 465 (2017).

7. S. J. M. Habraken, K. Stannigel, M. D. Lukin, P. Zoller, and P. Rabl, "Continuous mode cooling and phonon routers for phononic quantum networks," New J. Phys. 14, 115004 (2012).

8. G. Li, X. Xiao, Y. Li, and X. Wang, "Tunable optical nonreciprocity and a phonon-photon router in an optomechanical system with coupled mechanical and optical modes," Phys. Rev. A 97, 023801 (2018).

9. H. Zhang, J. Zhang, S. Sun, D. Wu, R. Zhao, R. Wang, and S. Dai, "Self-modelocking and self-phase modulation in Tm-doped double clad fiber laser for pulse peak power enhancement and multi-wavelength generation," Opt. Laser Technol. 141, 107128 (2021)

10. W. Luo, Y. Ren, J. Feng, X. Li, S. Lv, M. Qu, L. Jing, and X. Chen, "Threedimensional $\mathrm{Ag}_{2} \mathrm{~S}$ cubes for switchable multi-wavelength ultrashort pulse application," Nanotechnology 32, 355202 (2021).

11. P. Huang, X. Shu, and Z. Zhang, "Multi-wavelength random fiber laser with switchable wavelength interval," Opt. Express 28, 28686 (2020).

12. T. Zhai, X. Wu, F. Tong, S. Li, M. Wang, and X. Zhang, "Multi-wavelength lasing in a beat structure," Appl. Phys. Lett. 109, 261906 (2016).

13. B. Guo, X. Guo, L. Tang, W. Yang, Q. Chen, and Z. Ren, "Ultra-long-period grating-based multi-wavelength ultrafast fiber laser," Chin. Opt. Lett. 19, 071405 (2021)

14. Y. An, B. Sun, P. Wang, L. Xiao, H. Liu, and H. Xie, "A 1×20 MEMS mirror array with large scan angle and low driving voltage for optical wavelengthselective switches," Sensor. Actuat. A 324, 112689 (2021).

15. Y. Doi, T. Yoshimatsu, Y. Nakanishi, S. Tsunashima, M. Nada, S. Kamei, K. Sano, and Y. Ishii, "Receiver integration with arrayed waveguide gratings toward multi-wavelength data-centric communications and computing," Appl. Sci. 10, 8205 (2020).

16. H. W. Jones, M. K. Burdette, Y. Bandera, E. Zhang, I. K. Foulger, J. Binder, J. Weick, and S. H. Foulger, "Sequential intraparticle Förster resonance energy transfer for multi-wavelength bioimaging," Opt. Mater. Express 11, 1742 (2021).

17. M. Piniard, B. Sorrente, G. Hug, and P. Picart, "Theoretical analysis of surface-shape-induced decorrelation noise in multi-wavelength digital holography," Opt. Express 29, 14720 (2021).

18. C. Rao, L. Zhu, N. Gu, X. Rao, L. Zhang, H. Bao, L. Kong, Y. Guo, L. Zhong, X. Ma, M. Li, C. Wang, X. Zhang, X. Fan, D. Chen, Z. Feng, X. Wang, and $Z$. Wang, "A high-resolution multi-wavelength simultaneous imaging system with solar adaptive optics," Astron. J. 154, 143 (2017).

19. H. Gewiss, U. Timm, J. Kraitl, B. Brock, and H. Ewald, "Non-invasive multi wavelengths sensor system for measuring carboxy- and methemoglobin," Current Direct. Biomed. Eng. 3, 441 (2017).

20. M. Li, Y. Liu, F. Zhang, X. Zhang, Z. Zhang, A. A. A. Omer, S. Zhao, and W. Liu, "Design of multi-passband polymer multilayer film and its application in photovoltaic agriculture," Chin. Opt. Lett. 19, 112201 (2021).

21. H. Zhou, X. Jiang, J. Yang, Q. Zhou, T. Yu, M. Wang, and T. Yu, "Wavelength-selective optical waveguide isolator based on nonreciprocal ring-coupled Mach-Zehnder interferometer," J. Lightw. Technol. 26, 3166 (2008).

22. T. Huang, Y. Sun, and Z. Ouyang, "Numerical analysis of dual-wavelength nonreciprocal phase shifter for magneto-optical isolators on silicon-on-insulator system," Opt. Eng. 53, 117112 (2014).

23. Y. Hadad and B. Z. Steinberg, "Magnetized spiral chains of plasmonic ellipsoids for one-way optical waveguides," Phys. Rev. Lett. 105, 233904 (2010).

24. A. B. Khanikaev, S. H. Mousavi, G. Shvets, and Y. S. Kivshar, "One-way extraordinary optical transmission and nonreciprocal spoof plasmons," Phys. Rev. Lett. 105, 126804 (2010).
25. L. Bi, J. Hu, P. Jiang, D. H. Kim, G. F. Dionne, L. C. Kimerling, and C. A. Ross, "On-chip optical isolation in monolithically integrated non-reciprocal optical resonators," Nat. Photon. 5, 758 (2006).

26. M.-C. Tien, T. Mizumoto, P. Pintus, H. Kromer, and J. E. Bowers, "Silicon ring isolators with bonded nonreciprocal magneto-optic garnets," Opt. Express 19, 11740 (2011).

27. V. F. Nezhad, C. You, and G. Veronis, "Nanoplasmonic magneto-optical isolator," Chin. Opt. Lett. 19, 083602 (2021).

28. Y. Shoji, M. Ito, Y. Shirato, and T. Mizumoto, "MZI optical isolator with Siwire waveguides by surface-activated direct bonding," Opt. Express 20, 18440 (2012)

29. A. Alberucci and G. Assanto, "All-optical isolation by directional coupling," Opt. Lett. 33, 1641 (2008).

30. L. Fan, J. Wang, L. T. Varghese, H. Shen, B. Niu, Y. Xuan, A. M. Weiner, and M. Qi, "An all-silicon passive optical diode," Science 335, 447 (2012).

31. B. Anand, R. Podila, K. Lingam, S. R. Krishnan, S. S. S. Sai, R. Philip, and A. M. Rao, "Optical diode action from axially asymmetric nonlinearity in an all-carbon solid state device," Nano Lett. 13, 5771 (2013).

32. C. Wang, X.-L. Zhong, and Z.-Y. Li, "Linear and passive silicon optical isolator," Sci. Rep. 2, 674 (2012).

33. Z. Yu and S. Fan, "Complete optical isolation created by indirect interband photonic transitions," Nat. Photon. 3, 91 (2009).

34. M. S. Kang, A. Butsch, and P. S. J. Russell, "Reconfigurable light-driven optoacoustic isolators in photonic crystal fibre," Nat. Photon. 5, 549 (2011).

35. N. A. Estep, D. L. Sounas, J. Soric, and A. Alù, "Magnetic-free non-reciprocity and isolation based on parametrically modulated coupled-resonator loops," Nat. Phys. 10, 923 (2014).

36. D. L. Sounas, C. Caloz, and A. Alù, "Giant nonreciprocity at the subwavelength scale using angular momentum-biased metamaterials," Nat. Commun. 4, 2407 (2013).

37. D. L. Sounas and A. Alù, "Angular-momentum-biased nanorings to realize magnetic-free integrated optical isolation," ACS Photon. 1, 198 (2014).

38. Q. Wang, F. Xu, Z. Y. Yu, X.-S. Qian, X.-K. Hu, Y.-Q. Lu, and H.-T. Wang, "A bidirectional tunable optical diode based on periodically poled LiNbO," Opt. Express 18, 7340 (2010).

39. K. Fang, Z. Yu, and S. Fan, "Photonic Aharonov-Bohm effect based on dynamic modulation," Phys. Rev. Lett. 108, 153901 (2012).

40. S. Longhi, "Non-reciprocal transmission in photonic lattices based on unidirectional coherent perfect absorption," Opt. Lett. 40, 1278 (2015).

41. L. Yuan, S. Xu, and S. Fan, "Achieving nonreciprocal unidirectional singlephoton quantum transport using the photonic Aharonov-Bohm effect," Opt. Lett. 40, 5140 (2015).

42. J. H. Wu, M. Artoni, and G. C. La Rocca, "Non-Hermitian degeneracies and unidirectional reflectionless atomic lattices," Phys. Rev. Lett. 113, 123004 (2014).

43. B. Peng, S. K. Özdemir, F. Lei, F. Monifi, M. Gianfreda, G. L. Long, S. Fan, F. Nori, C. M. Bender, and L. Yang, "Parity-time-symmetric whispering-gallery microcavities," Nat. Phys. 10, 394 (2014).

44. H. Ramezani, T. Kottos, R. El-Ganainy, and D. N. Christodoulides, "Unidirectional nonlinear PT-symmetric optical structures," Phys. Rev. A82, 043803 (2010).

45. C. E. Rüter, K. G. Makris, R. El-Ganainy, D. N. Christodoulides, M. Segev, and D. Kip, "Observation of parity-time symmetry in optics," Nat. Phys. 6, $192(2010)$

46. D. W. Wang, H. T. Zhou, M. J. Guo, J.-X. Zhang, J. Evers, and S.-Y. Zhu, "Optical diode made from a moving photonic crystal," Phys. Rev. Lett. 110, 093901 (2013).

47. S. A. R. Horsley, J. H. Wu, M. Artoni, and G. C. La Rocca, "Optical nonreciprocity of cold atom Bragg mirrors in motion," Phys. Rev. Lett. 110, 223602 (2013).

48. J. Gea-Banacloche, Y.-Q. Li, S.-Z. Jin, and M. Xiao, "Electromagnetically induced transparency in ladder-type inhomogeneously broadened media: theory and experiment," Phys. Rev. A 51, 576 (1995).

49. G. W. Lin, Y. H. Qi, X. M. Lin, Y. P. Niu, and S. Q. Gong, "Strong photon blockade with intracavity electromagnetically induced transparency in a blockaded Rydberg ensemble,” Phys. Rev. A 92, 043842 (2015).

50. H. Wu, J. Gea-Banacloche, and M. Xiao, "Observation of intracavity electromagnetically induced transparency and polariton resonances in a dopplerbroadened medium," Phys. Rev. Lett. 100, 173602 (2008). 
51. S. Zhang, Y. Hu, G. Lin, Y. Niu, K. Xia, J. Gong, and S. Gong, “Thermalmotion-induced non-reciprocal quantum optical system," Nat. Photon. 12, 744 (2018).

52. K. Xia, F. Nori, and M. Xiao, "Cavity-free optical isolators and circulators using a chiral cross-Kerr nonlinearity," Phys. Rev. Lett. 121, 203602 (2018).

53. G. Lin, S. Zhang, Y. Hu, Y. Niu, J. Gong, and S. Gong, "Nonreciprocal amplification with four-level hot atoms," Phys. Rev. Lett. 123, 033902 (2019).

54. Y. Hu, S. Zhang, Y. Qi, G. Lin, Y. Niu, and S. Gong, "Multiwavelength magnetic-free optical isolator by optical pumping in warm atoms," Phys. Rev. Appl. 12, 054004 (2019).
55. S. Fan, Y. Qi, G. Lin, Y. Niu, and S. Gonga, "Broadband optical nonreciprocity in an N-type thermal atomic system," Opt. Commun. 462, 125343 (2020).

56. L. Yang, Y. Zhang, X.-B. Yan, Y. Sheng, C.-L. Cui, and J.-H. Wu, "Dynamically induced two-color nonreciprocity in a tripod system of a moving atomic lattice," Phys. Rev. A 92, 053859 (2015).

57. Y. Choi, C. Hahn, J. W. Yoon, S. H. Song, and P. Berini, "Extremely broadband, on-chip optical nonreciprocity enabled by mimicking nonlinear antiadiabatic quantum jumps near exceptional points," Nat. Commun. 8, 14154 (2016).

58. A. Seif and M. Hafezi, "Broadband optomechanical nonreciprocity," Nat. Photon. 12, 60 (2018). 